\title{
DIRECT ELECTRON ABSORPTION OF FAST WAVES ON THE DIII-D TOKAMAK
}

by

C.C. PETTY, R.I. PINSKGER, M. PORKOLAB, F.W. BAITY,** P. T. BONOLI, S.C. CHIU, J.S. deGRASSIE, R.H. GOULDING,** D.J. HOFFMAN, ${ }^{* *}$ R.A. JAMES, ${ }^{* * *}$ H. KAWASHIMA, JOHN LOHR, T.C. LUCE, M.J. MAYBERRY, and R. PRATER

This is a preprint of a paper to be presented at the Europhysics Topical Conference on RF Heating and Current Drive of Fusion Devices, July 7-10, 1992, Brussels, Belgium, and to be printed in the Proceedings.

Work supported by

U.S. Department of Energy Contracts DE-AC03-89ER51114, DE-AC03-78ET51013, DE-AC02-76-CHO-3073, and DE-AC05-84OR21400

\footnotetext{
* Massachusetts Institute of Technology ** Oak Ridge National Laboratory

*** Lawrence Livermore Nationai Laboratory

**** Japan Atomic Energy Research Institute
}

GENERAL ATOMICS PROJECT 3466 JUNE 1992

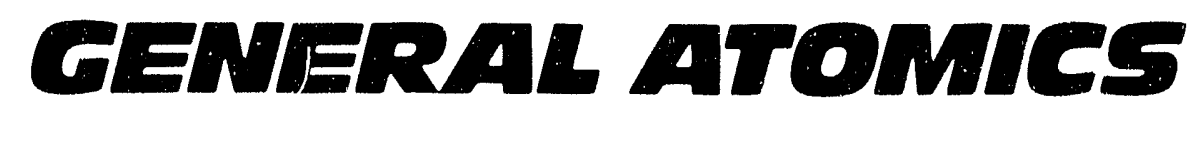

Mroun 


\title{
DIRECT ELECTRON ABSORPTION OF FAST WAVES ON THE DIII-D TOKAMAK*
}

\author{
C.C. FetTy, R.I. Pinsker, M. Porkolab, F. W. Baity, P. T. Bonoli, ${ }^{\dagger}$ \\ S.C. Chiv, J.S. deGrassie, R.H. Goulding, ' D.J. Hopfman, R.A. James, \\ H. KaWashima,' John Lohr, T. C. Luce, M.J. Mayberry, and R. Prater
}

General Atomics, San Diego, California, U. S.A.

Introduction. The fast Alfvén wave (FW) has previously been used to heat tokamak plasmas via ICRH ${ }^{1}$; however, FW can also be absorbed directly by electrons when the resonance condition $\omega-k_{1} v_{1 e}=0$ is satisfied. The direct electron absorption of $F W$ is due to electron Landau damping and transit-time magnetic pumping. ${ }^{2}$ For a Maxwellian electron distribution, the e-folding damping length for FW in the ICRF is given by ${ }^{3}$

$$
\lambda_{e}^{-1}=k_{\perp} R_{e} \frac{\sqrt{\pi}}{2} \beta_{e} \xi_{e} \exp ^{-\xi_{0}^{2}}\left(1+\frac{1}{a^{2}}\right),
$$

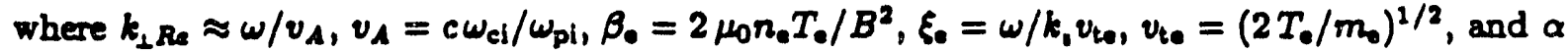
is a complicated function with $a^{-2}<1$ for this experiment. The FW absorption is maximized when $\xi_{0}=1 / \sqrt{2}$, which corresponds to a parallel index of refraction of

$$
\left|n_{1}\right| \equiv\left|k_{1}\right| c^{\prime} \omega=\sqrt{\frac{512}{T_{0}[\mathrm{keV}]}} .
$$

The FW absorption also decreases rapidly with increasing magnetic field since $\lambda^{-1} \propto B^{-3}$. The experiments on DIII-D vere aimed at chsacterizing the direct electron absorption of $F W$ as $\xi_{\text {. }}$ and $B$ were varied.

Experimental Setup. The FW heating experiments were conducted at $60 \mathrm{MHz}$ using a four-strap antenna with $(0, \pi, 0, \pi),(0,0,0,0)$, and $(0, \pm \pi / 2, \pm \pi, \pm 3 \pi / 2)$ phasings. The current straps are $45 \mathrm{~cm}$ high arii $11 \mathrm{~cm}$ wide, and are spaced $22.5 \mathrm{~cm}$ apart (center-to-center). The Faraday shield consists of two staggered tiers of Incenel rods which are copper plated and have a titanium carbonitride coating. The FW antenna is fed by a single $2 \mathrm{MW}, 30$ to $60 \mathrm{MHz}$ transmitter. F'or $(0, \pi, 0, \pi)$ phasing, the coupled parallel index of refraction was found to be $\left|n_{1}\right| \approx 9$, which is optimal for direct electron absorptivi at electron temperatures near $3 \mathrm{keV}$ after accounting for the upshift of the spectrum due to toroidal effects. For $(0, \pm \pi / 2, \pm \pi, \pm 3 \pi / 2)$ phasing, a coupled parallel index of refraction of $\left|n_{v}\right| \approx 5$ was measured, which requires $T_{e} \approx 11 \mathrm{keV}$ for optimal damping. The antenna end nearby vacuum vessel wall surfaces were coated with low- $Z$ materials (such as boron or carbon) for these experiments to reduce the metal influx and radiated power.

Experimental Results: Symmetric Phasing. Efficient direct electron heating by $F W$ injection bas been observed on the DIII-D tokainak, " with coupled power levels of up to $1.4 \mathrm{MW}$

\footnotetext{
*Rejiort of work sponsored by the U.S. Dlepartment of Energy under Contracts DE-AC03. 89ER51114, DE-AC03-78ET51013, DE-ACJ2-i5-CHO-3073, and DE-AC05-84OR21400.

'Permanent address: Massachusetts Institute of Technology, Cambridge, Massachusetts, U.S.A. 'Permanent address: Oak Ridge National Laboratory, Oak Ridge, Tennessee, U.S.A.

'Permanent address: Lawrence Livermore National Laboratory, Livermore, California, U.S.A.

'Permanent address: Japan Atomic Energy Research Institute, Tokai, Japan.

'Present address: Stanford University, Stanford, California, U.S.A.
} 
achieved. Plasma heating was observed by the increase in the central electron temperature (determined from Thomson scattering and ECE) and by the increase in the MHD and diamagnetic stored energies. The fraction of absorbed fast wave power is estimated from

$$
\eta=\frac{W / P}{\tau_{\text {ITER }}}
$$

where $W$ is the total stored energy, $P$ is the total inpui power, and $\tau_{\text {ITER }}$ is the ITER-89 power scaling relation developed for the ITER design":

$$
\tau_{\text {ITER }}=0.048 I^{0.85} R^{1.2} a^{0.3} \Sigma^{0.1} B_{\mathrm{T}}^{0.2}(A \kappa / P)^{0.5} .
$$

The fraction of absorbed fast wave power for a magnetic field scan is shown in Fig. 1 for doublenull-divertor plasmas. Although Eq. (1) predicts a low first pass damping of the FW and thus a strong $B_{\mathrm{T}}$ scaling of the absorption, the $\mathrm{FW}$ heating efficiency was $\approx 100 \%$ for all magnetic fields, indicating that multiple pass absorption of the FW may be occurring.

The FW heating experiments were performed in deuterium plasmas with a low hydrogen fraction $(\approx 2 \%)$. Since there was a second harmonic hydrogen resonance passing through the magnetic axis for $B_{\mathrm{T}}=2 \mathrm{~T}$, ion cyclotron absorption may compete with direct electron absorption at this magnetic field. However, full wave code calculations show that the direct electron damping is typically five times stronger than second harmonic hydrogen damping under these conditions. The experimental data do not exclude the possibility of this small amount of direct ion heating at $B_{\mathrm{T}}=2 \mathrm{~T}$; however, the data strongly indicate that the bulk of the plisma heating is due to direct electron absorption, for the following reasons. No dependence of thy plasma stored energy time on the location of the second hamonic hydrogen resonance was opserved during $B_{\mathfrak{T}}$ scans. Also, the rise time of the electron temperature is much shorter than the calculated slowing down time of an energetic ion tail on electrons. In addition, there is no evidence of a large perpendicular fast ion population from the diamagnetic loop measurements. Finally, the power deposition profile measured by modulating the fast wave power (discussed below) was centrally peaked and was independent of the location of the second harmonic cyclotron resonance for hydrogen.

The central electron heating by FW was found to be sensitive to the target electron temperature, in contrast to the absence of any observable $B_{\mathrm{T}}$ scaling of the fast wave absorption.

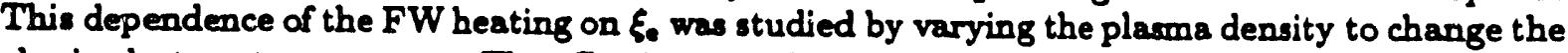
ohmic electron temperature. The effectiveness of FW electror heating increased substantially as the ahmic electron increased above $1 \mathrm{keV}$. This threshold corresponds to a ratio of the (toroj -ally down-shifted) wave parallel phase velocity to the electron thermal velocity of $\xi_{e} \approx 1.3$.

The FW absorption profile was measured directly by modulating the amplitude of the of power at $10 \mathrm{~Hz}$. By observing the response of the local electron temperature across tie plasma while the FW power was modulated, the power deposition profile was measured to be centrally peaked for $(0, \pi, 0, \pi)$ antenna phasing, as shown in Fig. 2(a). A fourier analysis of the ECE signals (cross-correlation with FW power) was used and the ECE channels were mapped to flux surfaces. The measured shape of the absorption profile is consistent with the deposition profile calculated for this experimental condition from a full wave code, shown in Fig. 2(b). The amplitude of the measured deposition profile is lower than the calculated value due to the lasge fraction of conductive losses at this relatively low modulation frequency. Also shown in Fig. 2(a) is the power absorption profile for $(0,0,0,0)$ phasing. As expected from earlier results, this low $\left|n_{1}\right|$ phasing was found to heat, very poorly since $\xi_{e} \rightarrow 1$ for this phasing.

Finally, $\mathrm{H}$-mode was obtained with FW heating alone for the first time in the direct electron heating regime $\left(\omega=8 \omega_{D}\right)$. The power threshold for H-mode with FW heating was somewhat lower than the power threshold for ECH or NBI heating. The normal H-mode characteristics 
were observed, such as a drop in the divertor light, increases in the density and stored energy, and broadening of the current profile. Quasi-steady-state ELMing H-mode periods as long as $1.35 \mathrm{sec}$ have been produced, and energy confinement time improvements of up to $\tau_{H} / \tau_{\mathrm{L}}=2.0$ have been achieved. H-modes have also been achieved with combined FW and NBI heating.

Experimental Results: Current Drive Phasing. Substantial electron heating and decreases in loop voltage were observed during FW injection with $(0, \pm \pi / 2, \pm \pi, \pm 3 \pi / 2)$ phasings at magnetic fields of $1.1 \mathrm{~T}$ and $2.0 \mathrm{~T}$, with or without electron preheating by $60 \mathrm{GHz} \mathrm{ECH}$. A manifest difference in electron heating and impurity influx between co-current and countercurrent phasing was observed for $B_{\mathrm{T}}=2.0 \mathrm{~T}$, with the counter-current plasing heating better and producing fewer impurities. When the plasma current was reversed, the effects of the antenna phasing reversed with it, so that the phasing counter to the new $I_{p}$ direction was again superior (see Fig. 3). The reason for this difference in electron heating is not presently understood. For this magnetic field, a perpendicular tail in the electron distribution function was seen by an $x$-ray spectrometer during $F W$ heating alone, with $T_{\text {tail }} \approx 8 \mathrm{keV}$. As shown in Fig. 4, co-current phasing produced a larger tail than counter-current phasing, suggesting that the residual toroidal electric field was aiding the of wave in creating the energetic tail. This electron tail may be due to the mode conversion (or direct coupling) of some of the FW power into IBWs, which are then absorbed by electrons through Landau damping.

For low density plasmas at $B_{\mathrm{T}}=1.1 \mathrm{~T}$, a peak electron temperature of $7 \mathrm{keV}$ was obtained while cverlapping $0.85 \mathrm{MW}$ of FW power with $0.64 \mathrm{MW}$ of ECH power for a short period. The energy confinement time for FW heating at this magnetic field was equivalent to the energy confinement time for ECH, indicating that the FW heating was efficient. An energetic electron tail created by ECH for this condition was enhanced during FW hesting, again with co-current phasing having a larger effect than counter-current phasing. The tail temperature was $\approx 4$ times greater than the central thermal temperature, and the energetic electron density was $\approx 10 \%$ of the central thermal density. No electron tail was seen during FW heating alone at $1 \mathrm{~T}$.

Conclusions. Efficient direct electron absorption of FW in the ICRF has been observed on the DIII-D tokamak. For symmetric phasings, the FW heating efficiency was independent of magnetic field, indicating multiple pass absorption. The central electron heating by the FW had a strong dependence on the target electron temperature. The power deposition profile was measured by modulating the FW power and was found to be peaked at the center. H-mode was obtained with FW heating alone for the first time in the direct electron heating regime. For current drive phasings, a manifest difference in electron heating and impurity influx between co-current and counter-current phasing was observed for $B_{T}=2.0 \mathrm{~T}$, with the counter-current phasing heating better and producing fewer impurities. For this magnetic field, an energetic tail in the electron distribution function was seen by an $x$-ray spectrometer during $F W$ heating alone, with co-current phasing producing a larger tail than counter-current phasing.

\section{References}

${ }^{1}$ D.G. Swanson, Phys. Fluids 28, 2645 (1985).

${ }^{2}$ T.H. Stix., Nucl. Fusion 15, 737 (1975).

${ }^{3}$ M. Porkolab, in Radiofrequency Power in Plasmas (Proc. 9th Top. Conf., Charleston, 1991), American Institute of Physics, New York (1992), p. 197.

'C.C. Petty et al., in Radjofrequency Power in Plasmas (Proc. 9th Top. Conf., Charleston, 1991), American Institute of Physics, New York (1992), p. 96.

BITER Team, "ITER Conceptual Design - Interim Repcrt," LAEA, Vienna (1989).

"P.T. Bonoli and M. Porkolab, in Radiofrequency Power" in Plasmas (Proc. 9th Top. Conf., Charleston, 1991), American Institute of Physics, New York (1992), p. 155. 


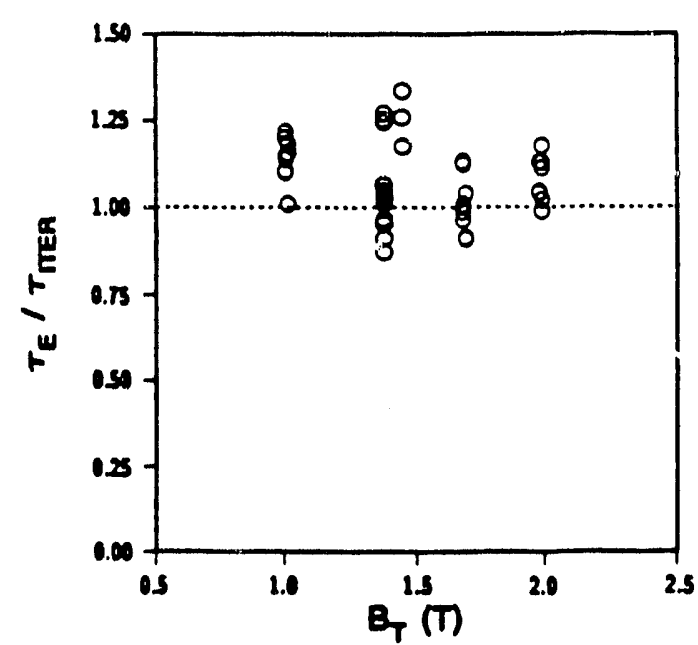

Frc. 1. Fraction of absorbed FW power for a magnetic field $\operatorname{scan}\left(I_{\mathrm{p}}=0.5 \mathrm{MA}\right.$, $\bar{n}_{\approx} \approx 1.6 \times 10^{19} \mathrm{~m}^{-3}$ ).
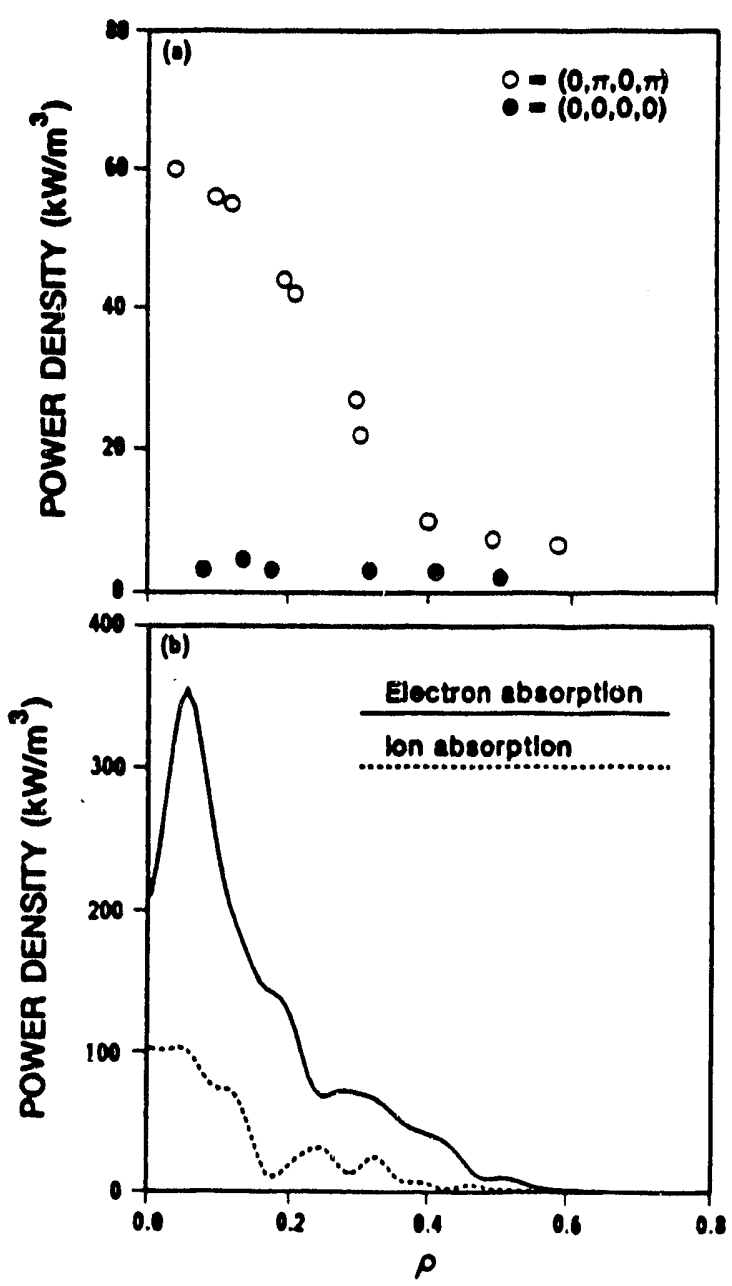

FIG. 2. (a) Absorbed FW power density versus normalized radius $(\rho)$ from modulation experiments $\left(I_{p}=1.0 \mathrm{MA}, B_{T}=2.0 \mathrm{~T}\right)$. (b) Power deposition profile from full wave code using 65 poloidal and 5 toroidal modes.

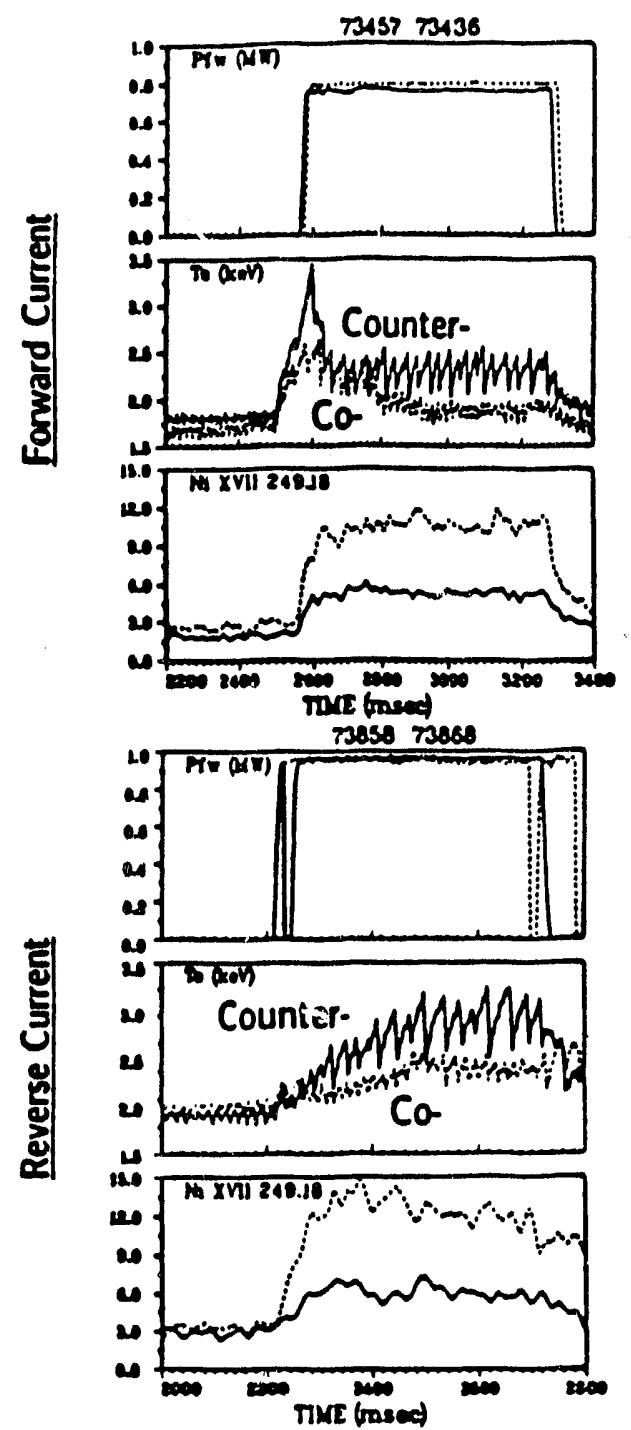

Fig. 3. Effect of co-current and countercurrent FW heating on electron temperature and impurity influx for forward and reverse $I_{p}$ directions $\left(I_{\mathrm{p}}= \pm 0.8 \mathrm{MA}, B_{\mathrm{T}}=2.0 \mathrm{~T}\right)$.

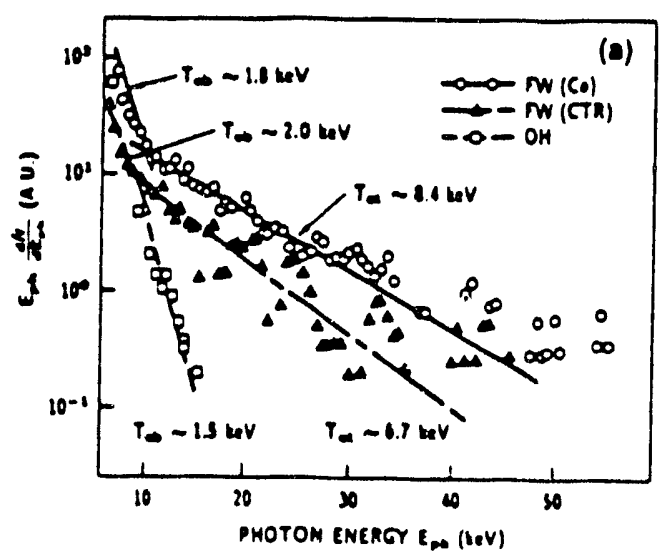

Fic. 4. X-ray energy spectra near the plasma center for co-current and counter-current FW heating $\left(I_{\mathrm{p}}=0.8 \mathrm{MA}, B_{\mathrm{T}}=2.0 \mathrm{~T}\right)$. 

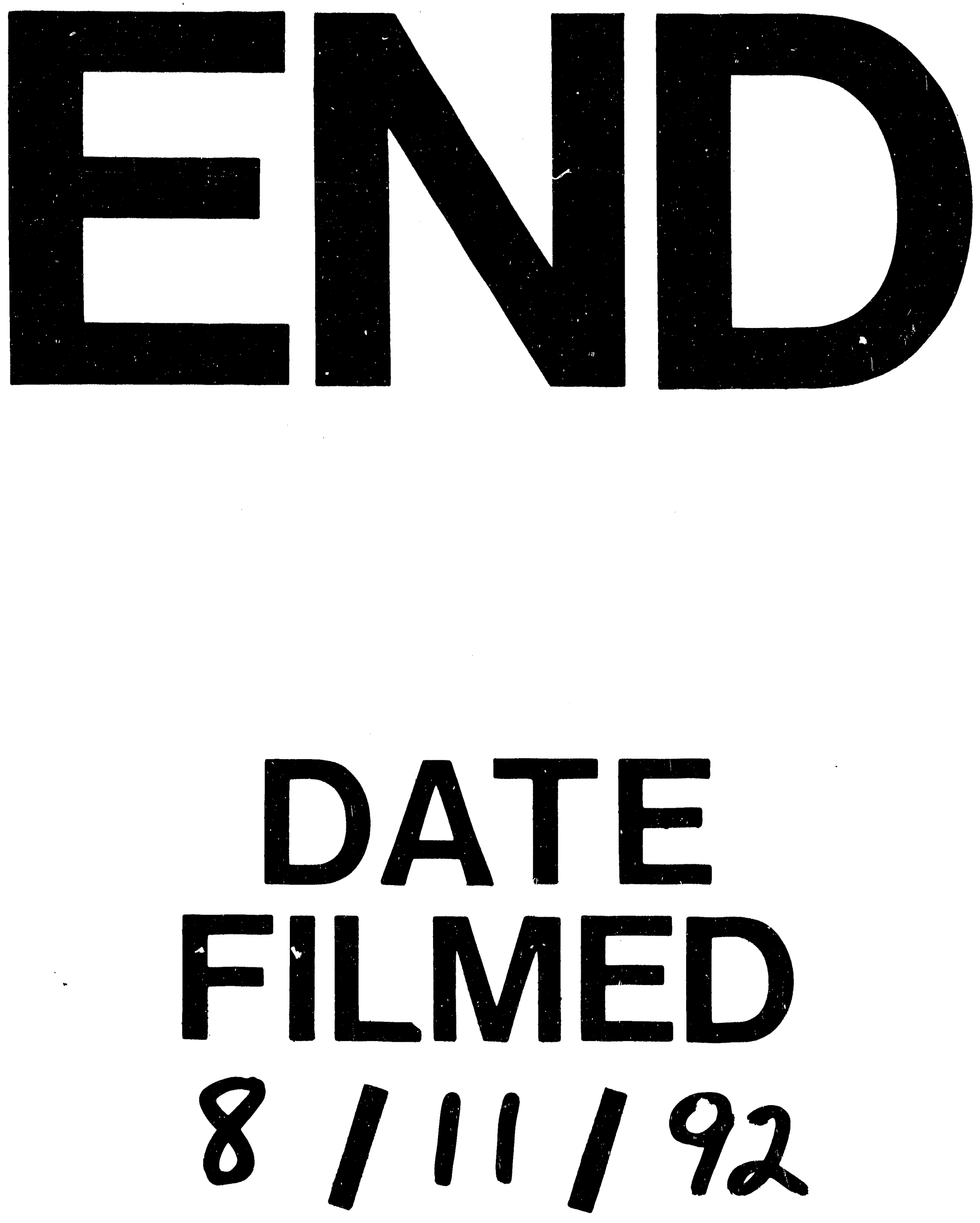
